DOI: 10.22403/UQROOMX/TYP19/05

\title{
La desincorporación, disminución de categoría o de superficie en áreas protegidas (fenómeno PADDD) y su efecto en el manejo de recursos forestales en un ejido del Nevado de Toluca, México
}

José Antonio de la Cruz-Hernández* Tecnológico de Estudios Superiores de Valle de Bravo

Víctor Ávila-Akerberg María Gladys Rivera Herrejón Ivonne Vizcarra Bordi Instituto de Ciencias Agropecuarias y Rurales Universidad Autónoma del Estado de México

\section{Resumen}

El presente artículo analiza el fenómeno PADDD (desincorporación, disminución de categoría o de superficie en áreas protegidas) y su efecto en el manejo de recursos forestales en un ejido del centro de México, con territorio en el área natural protegida Nevado de Toluca. La metodología empleada parte de una investigación documental en torno a los cambios en las políticas ambiental y forestal que incidieron sobre el territorio del ejido San Francisco Oxtotilpan desde principios del siglo xx hasta 2014; caracterizando los eventos que se pudieran calificar como acciones PADDD con base en la Guía Técnica PADDDtracker.org. Asimismo, se propuso el concepto de reversión para eventos que revierten procesos PADDD. Se seleccionaron 16 eventos como procesos PADDD, y se elaboró una matriz identificando su fecha, categoría PADDD, causa, área afectada y signo según su efecto sobre la conservación. Posteriormente se desarrolló otra matriz donde los eventos elegidos se clasificaron desde tres perspectivas: valoración PADDD, conservación como comanejo sustentable, o neopopulista, y conservación como exclusión de la población, o visión clásica; en una escala de muy bueno (4), bueno (2), neutro (0), malo (-2) y muy malo (-4). Esto fue graficado reconociendo tendencias y similitudes entre estas perspectivas. En conclusión, se aprecia la generación de políticas ambientales y forestales erráticas y muchas veces contradictorias en el Nevado de Toluca, además de la similitud entre las perspectivas PADDD y el manejo como exclusión de la población, y, en general, el antagonismo de estas con el comanejo sustentable.

\section{Palabras clave}

Nevado de Toluca, PADDD, areas naturales protegidas, manejo forestal comunitario, ejido San Francisco Oxtotilpan. 


\title{
Disincorporation, downgrading or downsizing in protected areas (PADDD phenomenon) and its effect on forest resource management in an ejido of the Nevado de Toluca, Mexico.
}

\begin{abstract}
This article analyzes the phenomenon PADDD (downgrading, downsizing and degazettement in protected areas) and its effect on the management of forest resources in an ejido (agrarian core) in the center of México, with territory in the protected natural area, Nevado de Toluca. The methodology employed part of documentary research on changes in forest and environmental policies that impacted on the territory of the ejido San Francisco Oxtotilpan since the beginning of the 20th century until 2014 year. Characterizing events that could qualify as PADDD actions based on the Guide technique PADDDtracker.org. It was also proposed the concept of "Reversión" for events that reverse processes PADDD. We selected 16 events as processes PADDD, elaborating a matrix identifying the date, category PADDD, cause, affected area and sign according to its effect on conservation. Subsequently developed another matrix where selected events are classified from three perspectives: that of "PADDD rating", of the conservation as sustainable co-management or "Neopopulista" and the conservation as exclusion of the population or "Classical view"; on a scale of very good (4), good (2), neutral (0), bad(-2) and very bad (-4). This was charted recognizing trends and similarities between these perspectives. As a result, it is appreciated the generation of erratic environmental and forestry policies and often contradictory in the Nevado de Toluca as well as the similarity between the PADDD perspectives and management as exclusion of the population, and in general the antagonism with the sustainable co-management.
\end{abstract}

\section{Key words}

Nevado de Toluca, PADDD, Protected areas, Community Forest Management, Ejido San Francisco Oxtotilpan.

*E-mail: vicaviak@gmail.com 


\section{Antecedentes}

En la actualidad, el mundo atraviesa una profunda crisis ambiental en la cual algunos de los principales umbrales de indicadores ambientales del estado del planeta ya han sido rebasados con efectos irreversibles para la biósfera (Rockström et al., 2009), lo que ha llevado a buscar estrategias globales y regionales que permitan disminuir la velocidad de estos cambios y la vulnerabilidad ante ellos.

Uno de los elementos principales que pueden ralentizar esta dinámica es la conservación de los bosques (PNUMA, 2012), sin embargo, la tasa de deforestación en el nivel mundial es de 16 millones de ha/año, mientras que México ocupa el sexto lugar en deforestación, con una pérdida anual de 69200 ha/año y conservando aún 64802000 ha de uso de suelo con vegetación forestal (FAO, 2010).

Entre las estrategias que se han promovido como acuerdos globales, tanto para impulsar la conservación de los bosques y la biodiversidad, como la prevención, mitigación y adaptación al cambio climático, están el establecimiento de áreas naturales protegidas (ANP) y la participación de la población local en su manejo (Secretariat of the Convention on Biological Diversity, 2014; PNUMA, 2012; Francis, 2008).

De forma paralela pero convergente, el manejo comunitario de los bosques se ha presentado como una opción para la conservación de las áreas naturales en zonas forestales y la biodiversidad que en ellas se encuentra (Rights and Resources Initiative, 2012), incluyendo en algunos casos ANP (Porter-Bolland et al., 2011).

En México, como producto del proceso revolucionario de principios del siglo xx, se propició la propiedad colectiva de terrenos forestales en gran parte del país (Klooster, 2003), lo cual se sumó, a finales de la década de los ochenta, a otros procesos sociales de defensa comunitaria, lo que llevó a la aparición de iniciativas para el surgimiento de la silvicultura y empresas forestales comunitarias (Conafor, 2015), siendo esta nación, en el plano mundial, pionera en estos procesos (Barton Bray y Merino Pérez, 2005; Conafor, 2011).

Por otro lado, en las zonas forestales de nuestro país, y en especial en los bosques templados, las políticas ambiental y forestal se han desarrollado con contradicciones y desencuentros, sin lograr una articulación y continuidad que permita una gestión forestal sostenible, ni una verdadera protección de las áreas naturales (Merino Pérez y Segura-Warnholtz, 2007; Bautista Calderón, 2007). 
El proceso PADDD (Protected Area Downgrading, Downsizing, and Degazettement), que en español podríamos conceptualizar como degradación de categoría, disminución y desincorporación de las ANP ya decretadas, ha sido estudiado recientemente en diferentes partes del mundo (Mascia et al., 2014), y se han encontrando patrones similares de afectación a estas áreas en todo el planeta. Al examinar tales procesos se han identificado las principales causas que los generan, las cuales se han agrupado en 13 categorías: silvicultura, minería, petróleo y gas, agricultura industrial, industrialización, infraestructura, reivindicaciones de tierras, asentamientos rurales, subsistencia, degradación, soberanía del desplazamiento, alojamiento de refugiados y planificación de la conservación (Mascia et al., 2014).

El cambio de decreto del Anp Nevado de Toluca, de parque nacional a área de protección de flora y fauna, ha originado una polarización en la opinión pública entre quienes consideran que se le disminuye la categoría de protección y por tanto pone en peligro la conservación de sus valores ambientales, y los que lo ven como la oportunidad para implementar un adecuado manejo forestal comunitario que permita el mejoramiento de la masa forestal a través de la participación de los dueños y poseedores de los bosques.

De esta manera, el cambio de decreto del Nevado de Toluca se puede analizar como un proceso PADDD, sin embargo, al abordar este fenómeno desde el ámbito de un núcleo agrario, los efectos sobre la conservación de la biodiversidad y de los bosques no son tan categóricos como al revisarlos únicamente desde la visión de los efectos sobre la superficie a proteger o sobre los valores que se establecen en el decreto de creación del anp. Incluso las interpretaciones acerca del efecto de las decisiones en política pública forestal y de conservación pueden ser antagónicas dependiendo de si la perspectiva es entender la conservación como el comanejo del área y recursos forestales entre la autoridad ambiental y forestal y las comunidades, o neopopulista, o como la exclusión del aprovechamiento de los recursos naturales que se encuentran en las ANp, o visión clásica (Alcorn, 2005), la cual fue el modelo inicial de los parques nacionales en Estados Unidos y luego se reprodujo en otros países, entre ellos México.

El presente trabajo examinó los efectos de 16 eventos administrativo-jurídicos que incidieron en el territorio del ejido San Francisco Oxtotilpan y su zona aledaña como procesos PADDD en las ANP federales: área de protección de flora 
y fauna Nevado de Toluca y área de protección de recursos naturales (APRN) Cuencas de los Ríos Valle de Bravo, Malacatepec, Tilostoc y Temascaltepec, así como el Anp estatal Santuario del Agua Corral de Piedra. Además se identificó su repercusión desde la perspectiva del manejo forestal comunitario o del comanejo de ANp basado en la participación de los habitantes locales.

La importancia de esta investigación radica en su contribución para superar el enfoque unidimensional que puede ofrecer el análisis del cambio de decreto como un fenómeno PADDD exclusivamente, pero también en cuanto a la visión de que al abrir los bosques al aprovechamiento forestal comunitario estos mejorarán de forma automática la calidad de su masa forestal y generarán un mayor desarrollo en los núcleos agrarios que habían tenido vedados sus bosques a tal aprovechamiento. Por tanto, esta revisión puede incidir en la discusión sobre la política pública en materia ambiental y forestal que actualmente se está llevando a cabo en torno al manejo del área de protección de flora y fauna Nevado de Toluca.

\section{Metodología}

El área de estudio corresponde al ejido San Francisco Oxtotilpan, ubicado en la parte central del país, en el Estado de México y el municipio de Temascaltepec, en el Eje Neovolcánico Transversal Mexicano (figura 1).

En cuanto a la regulación ambiental, el ejido se encuentra en las ANp federales: área de protección de flora y fauna Nevado de Toluca, por arriba de los 3000 msnm, y el Aprn Cuencas de los Ríos Valle de Bravo, Malacatepec, Tilostoc y Temascaltepec, por debajo de la mencionada cota; así como una pequeña porción al suroeste en el ANp estatal Santuario del Agua Corral de Piedra (figura 2).

La metodología empleada en el presente trabajo inició con investigación documental y de campo, mediante entrevistas sobre los cambios en las políticas ambiental y forestal que afectaron al ejido San Francisco Oxtotilpan desde la creación del parque nacional Nevado de Toluca hasta la fecha, identificando los eventos como acciones PADDD con base en la Guía Técnica PADDDtracker.org. Asimismo, se propuso el concepto de reversión cuando los procesos PADDD son eliminados o revertidos. De este modo, se registraron 16 eventos jurídico-administrativos caracterizados como PADDD, con los cuales se elaboraron dos matrices 
La desincorporación, disminución de categoría o de superficie en áreas protegidas (fenómeno PADDD) y su efecto en el manejo de recursos forestales en un ejido del Nevado de Toluca, México

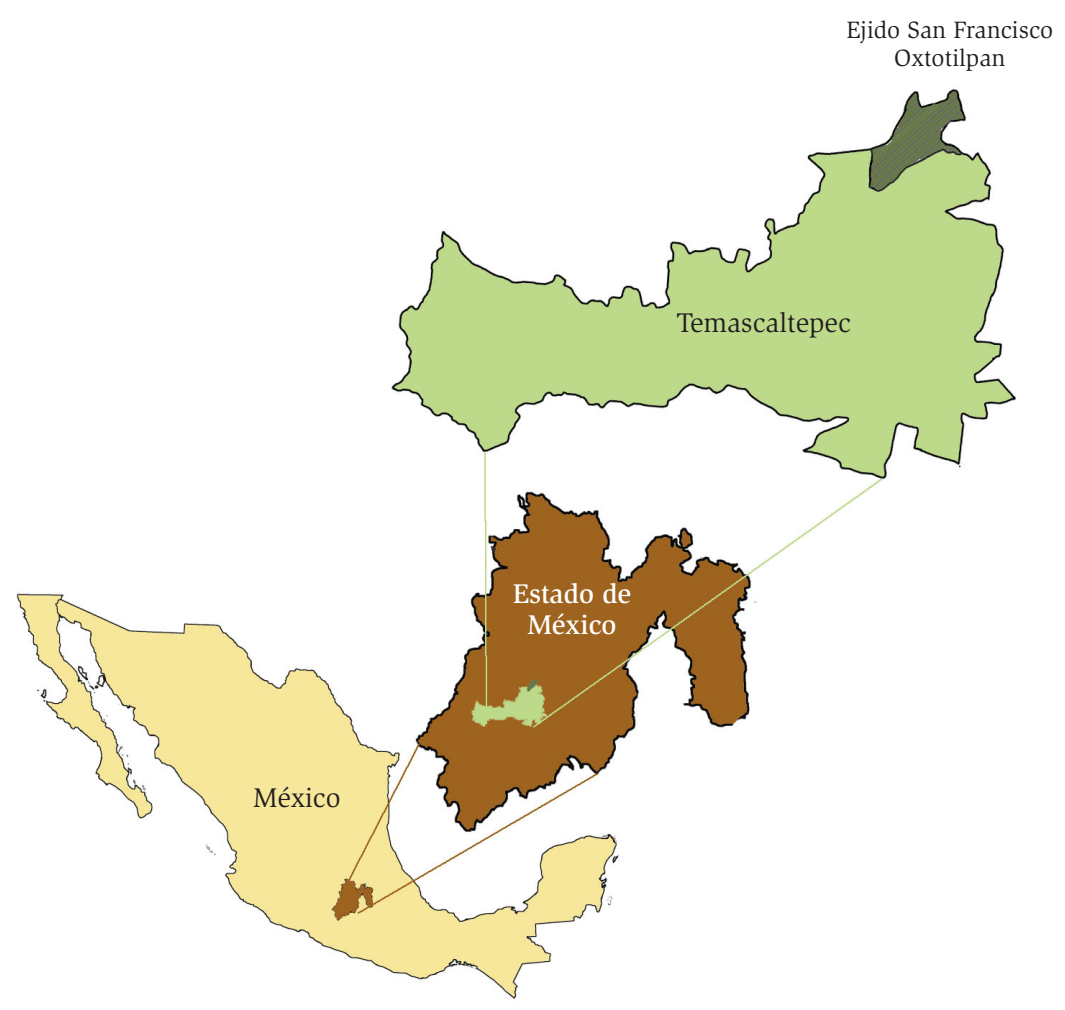

Figura 1. Ubicación de San Francisco 0xtotilpan

indicando su fecha, categoría PADDD, causa, área afectada y signo según su efecto sobre la conservación.

Posteriormente se realizó otra matriz donde cada evento se analizó desde tres perspectivas: valoración PADDD, conservación como comanejo sustentable y conservación como exclusión de la población.

La primera perspectiva es planteada por Mascia et al. (2014) desde el análisis de la disminución del grado de protección, tamaño o desaparición del régimen de protección en las ANP que se ha presentado en muchas áreas del mundo y que el Fondo Mundial para la Naturaleza ha documentado desde 2009 (Mascia et al., 2014), contrario al sentido común que lleva a pensar que, una vez decretada, un área protegida permanece inalterable en su superficie o 


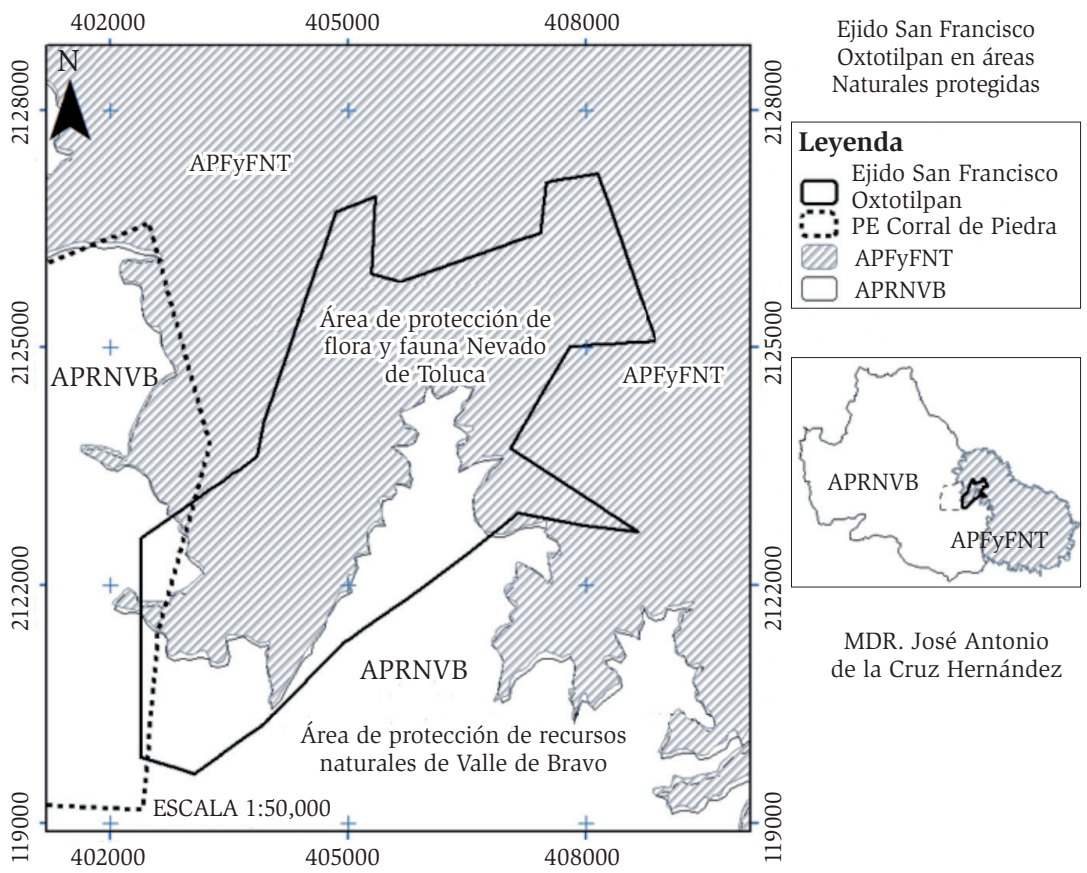

Figura 2. Ubicación de San Francisco 0xtotilpan en áreas naturales protegidas

régimen de protección. La perspectiva de conservación como comanejo sustentable, o neopopulista, propone que la población local participa en su manejo y se permite el aprovechamiento de recursos de manera sustentable, siendo posible alcanzar más fácil y permanentemente la conservación de sus valores ambientales (Alcorn, 2005). Por último, la perspectiva de conservación como exclusión de la población, o visión clásica, sostiene que la conservación de las áreas naturales requiere evitar la presencia de los seres humanos y el aprovechamiento de los recursos naturales mediante la vigilancia y restricción del acceso a las mismas, salvo en algunas áreas con fines de investigación o turismo (Alcorn, 2005).

Para calificar estos eventos se construyó una escala simple basada en evidencia de estudios sociales relacionados con el sector forestal y ambiental en México y el Estado de México, así como informes y documentos de trabajo de las áreas protegidas (Bautista Calderón, 2007; Cardeña Rodríguez, 2000; Conanp, 2013, 2014a y 2014b; Jujnovsky, Galván y Mazari-Hiriart, 2013; Merino Pérez, 
2004; Merino Pérez y Segura-Warnholtz, 2007; Klooster, 2007), de manera análoga a la metodología utilizada en estudios de medicina basada en evidencias (Manterola y Zavando, 2009). Al impacto de cada evento sobre el territorio se le asignó un valor cuantitativo desde cada perspectiva en una escala de muy bueno (4), bueno (2), neutro (0), malo (-2) y muy malo (-4). Los valores fueron asignados de acuerdo con los criterios presentados en el cuadro 1.

\section{Cuadro 1. Escala de categorías de impacto del evento.}

\begin{tabular}{llc} 
CATEGORÍA & DESCRIPCIÓN DEL IMPACTO DEL EVENTO & VALOR \\
Muy malo & $\begin{array}{l}\text { Generó procesos negativos irreversibles o de largo plazo } \\
\text { para los bosques o los valores del área natural protegida. }\end{array}$ & -4 \\
\hline \multirow{2}{*}{ Malo } & $\begin{array}{l}\text { Generó procesos negativos para los bosques o los } \\
\text { valores del área natural protegida con repercusiones en } \\
\text { el corto o mediano plazo. }\end{array}$ & -2 \\
\hline Nulo & No tuvo repercusiones reales para los valores del área. & 0 \\
Bueno & $\begin{array}{l}\text { Generó procesos positivos para bosques o los valores } \\
\text { del área natural protegida con repercusiones en el corto }\end{array}$ & 2 \\
\hline \multirow{2}{*}{ Muy bueno } & $\begin{array}{l}\text { Genera procesos positivos de largo plazo o permanentes } \\
\text { para los bosques o los valores del área natural protegida. }\end{array}$ & 4 \\
\hline
\end{tabular}

Fuente: Elaboración propia.

La categoría de calificación desde la perspectiva del PADDD y de la visión clásica de la conservación considera el valor dependiendo si la decisión originó procesos que llevaron a disminuir o aumentar la cobertura forestal o superficie del área, o a cambiar la normatividad permitiendo actividades que condujeran a la deforestación o degradación de los bosques. Por otro lado, desde la perspectiva neopopulista se calificó al evento con base en su influencia en la generación de una más elevada cultura forestal o apropiación de los bosques por parte de los ejidatarios, suponiendo que esta llevaría a una mayor protección por parte de sus usuarios al tener un mayor valor de uso del territorio y su patrimonio natural.

Esto fue graficado tomando en el eje de las "X" la serie de eventos en su secuencia temporal y en el de las "Y" el valor del evento para la perspectiva, reconociendo así las tendencias y similitudes entre las tres visiones. 


\section{Resultados}

En México, las políticas públicas de fomento y conservación de los recursos forestales y la creación y administración de ANp han tenido constantes vaivenes, así como en muchos casos duplicidad, ambigüedad y contradicción en las mismas. Para comprenderlo, se registraron, con base en información documental y entrevistas en campo, 16 eventos jurídico-administrativos que debieron incidir en el manejo de los recursos forestales del actual ejido San Francisco Oxtotilpan.

Estos eventos se identificaron a partir de la creación del parque nacional Nevado de Toluca en 1936, hasta su reciente recategorización como área de protección de flora y fauna. También se consideró la creación y recategorización de la zona protectora forestal área constitutiva de las Cuencas de los Ríos Valle de Bravo, Malacatepec, Tilostoc y Temascaltepec, la cual se sobrelapó con la zona oeste del parque nacional Nevado de Toluca, hasta su recategorización. Asimismo, en 2003 se instituyó dentro de su polígono el parque estatal Santuario del Agua Corral de Piedra. A continuación se presentan de manera resumida estos eventos.

\section{Eventos jurídico-administrativos en materia forestal y de conservación que incidieron en el ejido San Francisco Oxtotilpan}

1936. Creación del parque nacional Nevado de Toluca. El 25 de enero de 1936 el presidente Lázaro Cárdenas decreta el parque nacional Nevado de Toluca, con el objetivo de protegerlo contra la degradación, para mantener o restaurar los bosques, además de asegurar el abasto constante de agua necesaria para la agricultura e industria y mantener su belleza natural. Fue delimitado por la cota de los $3000 \mathrm{msnm}$, "salvando las porciones de terrenos agrícolas en cultivo y poblados que se encuentren dentro de la misma curva, a los que se les dará un radio de protección de 100 metros...”, con la instrucción de que la Secretaría de Hacienda y Crédito Público afrontara la indemnización de la expropiación, teniendo un plazo de seis meses los afectados para documentar su propiedad (Conanp, 2013: 5). Esta acción buscaba poner fin al severo deterioro que había sido producido por la explotación de empresas concesionarias que sin criterios dasonómicos y de modo irracional habían aprovechado los bosques de la región, sumado a la deforestación generada para saquear la madera utilizando como cubierta los reclamos legítimos para la dotación de tierra dentro 
del proceso de reforma agraria (Sánchez et al., 1990). Sin embargo, en general, las ANP creadas durante las tres primeras cuartas partes del siglo xx no operaron en realidad, pues las medidas y estrategias de protección eran prácticamente nulas, lo que contribuyó a una rápida degradación de estas áreas (Jujnovsky, Galván y Mazari-Hiriart, 2013).

1937. Creación de la reserva forestal Nevado de Toluca. Se determina una porción de terrenos para constituir una reserva forestal nacional, cuyos productos maderables, trabajados en forma racional y bajo la inmediata atención del Departamento Forestal y de Caza y Pesca, pudieran prestar los beneficios de orden económico indispensables a los grupos de trabajadores de la comarca, sin que con ello perjudicara la finalidad principal que se tuvo en cuenta para la expedición del decreto que establece el citado parque nacional. La reserva forestal se instituyó para paliar -de alguna manera- las demandas sociales por el aprovechamiento de los recursos forestales (Conanp, 2013).

1937. Dotación de tierras para conformar el ejido San Francisco Oxtotilpan. Se dotan 2270 hectáreas como ejido a campesinos de San Francisco Oxtotilpan y se ejecuta el 28 de junio de 1940 (Sedatu, s/f). De estas, 1570 se ubican dentro del área decretada parque nacional (Fucader y García Tepexpa, 2011). Esto como parte de un proceso de dotación de tierra que venía dándose en la región desde mediados de los veinte, hasta varios años posteriores al decreto de creación del parque nacional Nevado de Toluca (Conanp, 2013).

1941. Creación de la zona protectora forestal Cuencas de los Ríos Valle de Bravo, Malacatepec, Tilostoc y Temascaltepec. A fin de garantizar el abastecimiento de agua para la generación de energía eléctrica se elabora el proyecto de decreto para declarar zona protectora forestal los terrenos constitutivos de las Cuencas de los Ríos Valle de Bravo, Malacatepec, Tilostoc y Temascaltepec en el Estado de México con el propósito de "aumentar el caudal utilizable por la Comisión Federal de Electricidad, para lo cual se hace necesario limitar la tala inmoderada de los bosques, que ha ocasionado una disminución considerable de las aguas permanentes de estos..." (Conanp, 2014b: 8). Sin embargo, no cuenta con límites claramente establecidos y se sobrelapa con el parque nacional Nevado de Toluca y la reserva forestal. Además, como ya se mencionó, en realidad 
no operaron, ya que las medidas y estrategias de protección eran prácticamente nulas, lo que favoreció la rápida degradación de estas áreas (Jujnovsky, Galván y Mazari-Hiriart, 2013).

1947. Establecimiento de veda en bosques del Estado de México. El 29 de marzo de 1947 se publicó un decreto presidencial que, salvaguardando los derechos de las unidades industriales de explotación forestal que se habían concesionado a compañías papeleras en la Cuenca de México, estableció la necesidad de suspender por completo las explotaciones forestales, porque, según el mismo decreto, la tala inmoderada de los bosques había determinado un cambio en el régimen climático de la Mesa Central, afectando gravemente la precipitación fluvial. Por lo anterior se dispuso una veda ilimitada de recuperación aplicable a los bosques comprendidos dentro de los límites del Estado de México y del Distrito Federal. Pese a ello, se señala que la deforestación no se detuvo, pues la Secretaría de Agricultura continuó dando concesiones forestales que se justificaban con cortas culturales y como parte del cultivo de las masas forestales (Sánchez et al., 1990), y no se tenía la capacidad operativa institucional para vigilar el cumplimiento de estas vedas (Bautista Calderón, 2007). Por otro lado, los dueños y poseedores de los bosques fueron excluidos de la toma de decisiones sobre el aprovechamiento forestal, lo que generó inconformidad y desinterés hacia esta actividad. La veda fue levantada en 1970 (Sánchez et al., 1990).

1969. Creación de Protinbos. Pese a la veda forestal establecida en 1947, los bosques del Estado de México presentan un severo deterioro, por lo que el Gobierno del Estado de México toma la decisión de participar directamente en el manejo, protección, fomento e industrialización de los recursos forestales de la entidad, para lo cual instituye un organismo público descentralizado denominado Protectora e Industrializadora de Bosques (Protinbos). Nuevamente los dueños y poseedores son excluidos de las decisiones sobre el manejo forestal de sus predios (Sánchez et al., 1990).

1970. Levantamiento de veda en el Estado de México. El gobierno federal considera que con la instauración de Protinbos en el Estado de México se dan las condiciones para el cambio de una política forestal sustituyendo el proteccionismo, que no funcionó, por una buena administración del recurso forestal, 
por lo que deroga la veda a fin de que los poseedores de títulos de dominio de bosques en el Estado de México puedan celebrar contratos con la recién creada Protinbos. Asimismo, en 1971, en San Francisco de los Ranchos, Temascaltepec, aledaño a San Francisco Oxtotilpan, Protinbos establece un aserradero con objeto de beneficiar la materia prima forestal (Sánchez et al., 1990); sin embargo, la operación de este organismo marginó a los dueños y poseedores de los recursos forestales del manejo silvícola, por lo cual no se generó experiencia en los núcleos agrarios en la administración y operación de la producción, así como en la comercialización de la materia prima forestal (Cardeña Rodríguez, 2000).

1990. Creación de Probosque. Como parte del proceso de reestructuración de organismos auxiliares y fideicomisos del Gobierno del Estado, Protinbos fue desincorporada con el propósito de conformar una organización que tuviera como objetivo principal la protección y cuidado de los bosques; así, el 13 de junio de 1990, mediante el Decreto 124, se creó el organismo público descentralizado denominado Protectora de Bosques del Estado de México (Probosque), cuyo objeto y actividades se orientaron a la protección, conservación, reforestación, fomento y vigilancia de los recursos forestales del Estado de México (Gobierno del Estado de México-Secretaría del Medio Ambiente, s/f).

1991-1995. Nueva veda en el Estado de México. El 8 de mayo de 1991 el gobierno federal decreta la veda forestal y parcial en el Estado de México con respecto a las especies forestales en todas sus variedades, ubicadas dentro de los límites de la entidad. Esta veda se plantea debido a los altos índices de deforestación presentados. Se establece que la veda sería por el tiempo necesario para expedir las declaratorias de usos, reservas y destinos, con base en los programas de manejo integral de los recursos forestales en las cuencas y subcuencas hidrográficas del Estado de México, con el objetivo de delimitar los terrenos forestales que debían permanecer como tales, los que debían incorporarse al uso forestal, los que fueran susceptibles de ser utilizados en actividades agropecuarias y aquellos que debían permanecer inalterables (Poder Ejecutivo, 1991). Según un nuevo decreto del 26 de junio de 1995, para 1994 un grupo interinstitucional, junto con los sectores social y privado, llevaron a cabo los trabajos del Inventario Forestal Nacional, en la parte correspondiente al Estado de México, los cuales concluyeron que era posible realizar una explotación ordenada y racional de los 
recursos forestales de la entidad y que se habían logrado avances significativos en materia de conservación de suelos, protección de cuencas hidrográficas y reforestación, que habían permitido disminuir la alteración y los cambios del ambiente y del ciclo hidrológico, por lo que era posible levantar la veda temporal y parcial (Poder Ejecutivo, 1995).

1996. Cambios en la Ley General del Equilibrio Ecológico y Protección al Ambiente en su artículo 50 que habla sobre los parques nacionales. Se modifica el artículo 50 de la Ley General del Equilibrio Ecológico y Protección al Ambiente restringiendo el aprovechamiento forestal en parques nacionales. Antes de esta reforma se podían otorgar autorizaciones para realizar aprovechamientos forestales cuando existiera dictamen técnico de la secretaría que estableciera la conveniencia ecológica del aprovechamiento de que se tratara y dándose preferencia a quienes ahí habitaran en el momento de la expedición de la declaratoria respectiva (Poder Ejecutivo, 1988). Esto tuvo repercusiones sobre las posibilidades de desarrollo de los núcleos agrarios poseedores de estos terrenos forestales limitando acciones para su aprovechamiento y generando el desinterés en su protección y manejo.

1997. Autorización del Primer Programa de Manejo Forestal del Ejido San Francisco Oxtotilpan. Con los cambios en la Ley Forestal de 1986, que anularon el sistema de concesiones forestales, se reconocía el derecho de las comunidades a aprovechar directamente sus bosques y se proscribía el rentismo forestal, y los de 1992 abrían el mercado de los servicios técnicos forestales (Merino Pérez, 2004). Con el término de las vedas forestales se producen las condiciones para que en 1997 el ejido de San Francisco Oxtotilpan logre la autorización de su Primer Programa de Manejo Forestal con un ciclo de corta de diez años y una posibilidad de corta de $1662.99 \mathrm{~m}^{3}$ VTA en 380.5 ha en la zona del ejido fuera del parque nacional Nevado de Toluca.

2003. Creación del parque estatal Santuario del Agua Corral de Piedra. Se instituye el ANp parque estatal Santuario del Agua Corral de Piedra con el fin de "contribuir al desarrollo ambiental sustentable y a su vez, incrementar el nivel económico de los pobladores de la región; conservar los ecosistemas hidrológicos y forestales, en beneficio de la diversidad biológica; favorecer la recarga de 
los acuíferos y fomentar el desarrollo ecoturístico y la cultura del uso integral del recurso agua, suelo y de su flora y fauna” (Poder Ejecutivo del Estado de México, 2003); esta abarca una franja mínima del territorio del ejido San Francisco Oxtotilpan en su límite oeste. En el decreto se prohíbe la tala de arbolado en las zonas de protección y conservación, teniendo el ejido áreas con esta zonificación.

2005. Creación del área de protección de recursos naturales Valle de Bravo, Malacatepec, Tilostoc y Temascaltepec. Mediante un acuerdo presidencial se recategoriza la zona protectora forestal a los terrenos constitutivos de las Cuencas de los Ríos Valle de Bravo, Malacatepec, Tilostoc y Temascaltepec, en cuanto APRN con el fin de adecuarla como Anp dentro de las categorías vigentes desde 1995 en la Ley General del Equilibrio Ecológico y Protección al Ambiente. Esta área se traslapa con la reserva de la biósfera Mariposa Monarca y Bosencheve en su parte noroeste, con el parque nacional Nevado de Toluca en su zona este y envuelve por completo al Santuario del Agua Corral de Piedra. De esta manera, el ejido San Francisco Oxtotilpan queda inmerso en ANP, pues de las 270 ha que lo componen (García, 2008), 1570 ha caen dentro del parque nacional Nevado de Toluca y 700 ha se ubican únicamente en el APRN; en esta área es posible realizar el aprovechamiento forestal persistente siempre y cuando se elabore y autorice su programa de manejo forestal sustentable y su respectiva manifestación de impacto ambiental.

2007. Publicación del Programa de Conservación y Manejo del Parque Estatal Santuario del Agua Corral de Piedra. El Gobierno del Estado de México publica el Programa de Conservación y Manejo del Parque Estatal Santuario del Agua Corral de Piedra, el cual tiene por objetivo general "Proteger, conservar y restaurar el Parque Estatal Santuario del Agua Corral de Piedra mediante el establecimiento de bases de planeación con la finalidad de dar un aprovechamiento sustentable a los recursos físicos, biológicos y humanos" (Poder Ejecutivo del Estado de México, 2007). Sin embargo, en este documento no se identifica que el ejido San Francisco Oxtotilpan cuente con territorio dentro del ANP, aun cuando sí tiene una franja dentro del área sureste del polígono. El documento menciona en su zonificación que los permisos de aprovechamiento forestal otorgados antes del decreto serán respetados en todas sus zonas. 
2008. Autorización del Programa de Manejo Forestal de San Francisco Oxtotilpan ciclo 2008-2018. Se autoriza el Programa de Manejo Forestal para el Aprovechamiento de Recursos Forestales Maderables (Nivel Avanzado) del Ejido San Francisco Oxtotilpan, de acuerdo con el cual se propone una posibilidad de corta de $18891.177 \mathrm{~m}^{3}$ VTA, en 380.5 ha fuera del Anp Nevado de Toluca. Para lograr esta autorización, se tuvo que presentar una manifestación de impacto ambiental al encontrarse dentro del APRN. Cabe mencionar que, con base en los análisis dasonómicos realizados para el segundo programa de manejo forestal, se demostró que la calidad de la masa forestal y el volumen aprovechable se incrementaron.

2013. Recategorización del parque nacional Nevado de Toluca como área de protección de flora y fauna. El $1^{\circ}$ de octubre de 2013 se publicó en el Diario Oficial de la Federación el Decreto que reforma, deroga y adiciona diversas disposiciones del diverso publicado el 25 de enero de 1936, por el que se declaró Parque Nacional la montaña denominada "Nevado de Toluca" que fue modificado por el diverso publicado el 19 de febrero de 1937, otorgándole la categoría de área de protección de flora y fauna. Las áreas de protección de flora y fauna se constituyen en lugares que contienen los hábitats de cuyo equilibrio y preservación depende la existencia, transformación y desarrollo de especies de flora y fauna silvestres, y aunque en tales zonas se puede autorizar el aprovechamiento de recursos naturales, esta categoría de protección permite el establecimiento de modalidades para este tipo de aprovechamientos, con la finalidad de preservar las especies de flora y fauna que son objeto de protección, así como los hábitats donde estas se desarrollan (Conanp, 2014a).

\section{Análisis de eventos PADDD}

Los 16 eventos identificados relacionados con el fenómeno PADDD fueron calificados en las tres categorías planteadas por Mascia et al. (2014) para el análisis de este tipo de procesos:

- Degradación (Downgrade)

- Disminución (Downsize)

- Desincorporación (Degazette) 
La desincorporación, disminución de categoría o de superficie en áreas protegidas (fenómeno PADDD) y su efecto en el manejo de recursos forestales en un ejido del Nevado de Toluca, México

Las causas propuestas son silvicultura, minería, petróleo y gas, agricultura industrial, industrialización, infraestructura, reivindicaciones de tierras, asentamientos rurales, subsistencia, degradación, soberanía del desplazamiento, alojamiento de refugiados y planificación de la conservación.

Asimismo, se utiliza el concepto de reversión cuando los procesos PADDD son eliminados o se toman decisiones para la recuperación de las características originales del área en cuanto a categoría de protección y manejo de sus recursos de acuerdo con el decreto o tamaño del área. En el fenómeno de reversión se agregó la categoría "Protección" como causa de la misma, que tiene una connotación positiva. En el cuadro 2 se califican estos eventos en función de sus características principales.

Cuadro 2. Caracterización de eventos PADDD

\begin{tabular}{|c|c|c|c|c|c|}
\hline EVENTO & FECHA & $\begin{array}{c}\text { CATEGORÍA } \\
\text { PADDD }\end{array}$ & CAUSA & $\begin{array}{c}\text { ÁREA AFECTADA } \\
(\mathrm{HA})\end{array}$ & $\begin{array}{l}\text { SIGNO } \\
\text { PADDD }\end{array}$ \\
\hline
\end{tabular}

Cambios en la Ley

General del Equilibrio

Ecológico y Protección

al Ambiente limitan

aprovechamiento forestal

en parques nacionales

Primer aprovechamiento forestal persistente con un ciclo de corta de diez años en el ejido San Francisco Oxtotilpan

Creación del parque estatal Santuario del Agua Corral de Piedra

Creación del área de protección de recursos naturales Valle de Bravo, Malacatepec, Tilostoc y Temascaltepec
1997
Degradación
Silvicultura
$381 \mathrm{ZPF}$

1996 Reversión Protección Toda el área

Publicación del Programa de Conservación y Manejo Parque Estatal Santuario del Agua Corral 2003 Degradación Planificación

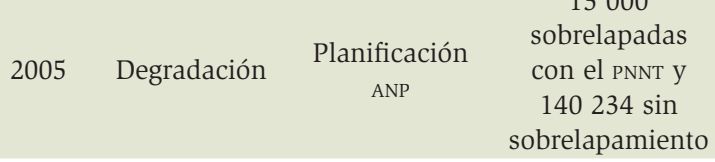

2007 Degradación $\quad 3622$
de Piedra 
Cuadro 2. Caracterización de eventos PADDD (finaliza)

\begin{tabular}{|c|c|c|c|c|c|}
\hline EVENTO & FECHA & $\begin{array}{l}\text { CATEGORÍA } \\
\text { PADDD }\end{array}$ & CAUSA & $\begin{array}{c}\text { ÁREA AFECTADA } \\
(\mathrm{HA})\end{array}$ & $\begin{array}{l}\text { SIGNO } \\
\text { PADDD }\end{array}$ \\
\hline $\begin{array}{l}\text { Autorización de Plan de } \\
\text { Manejo Forestal de San } \\
\text { Francisco Oxtotilpan ciclo } \\
2008-2018\end{array}$ & 2008 & Degradación & Silvicultura & 381 en APRN & - \\
\hline $\begin{array}{l}\text { Recategorización del } \\
\text { parque nacional Nevado } \\
\text { de Toluca a área de } \\
\text { protección de flora y } \\
\text { fauna }\end{array}$ & 2013 & Degradación & $\begin{array}{c}\text { Planificación } \\
\text { ANP }\end{array}$ & 53590 & - \\
\hline
\end{tabular}

Fuente: Elaboración propia.

\section{Análisis desde las perspectivas PADDD, neopopulista y clásica de la conservación}

Al tener en cuenta que la conceptualización del fenómeno PADDD es muy esquemática, se hizo un análisis calificando estos eventos desde las tres perspectivas ya mencionadas en la metodología:

- El fenómeno PADDd tomado de forma rigurosa considerando como negativos la disminución de superficie del ANP, el cambio de categoría, la permisividad de actividades de uso de recursos forestales y del territorio en actividades ajenas a la preservación de los valores del área; así como evaluando cambios en la legislación que afecten positiva o negativamente los valores de conservación.

- La conservación de los recursos forestales concebida como un comanejo entre las agencias y los dueños o poseedores de los recursos forestales para su aprovechamiento sustentable y que permite una mayor apropiación comunitaria de los bosques.

- La conservación entendida como la no alteración por actividades humanas de los valores biológicos y paisajísticos del ANP.

De esta manera, se generó la matriz representada en el cuadro 3, a partir de la cual se graficaron los eventos de acuerdo con su valor y secuencia en el tiempo. Para clarificar más su orientación o perspectivas se generó una línea de tendencia para cada visión (figura 3). 
La desincorporación, disminución de categoría o de superficie en áreas protegidas (fenómeno PADDD) y su efecto en el manejo de recursos forestales en un ejido del Nevado de Toluca, México

Cuadro 3. Evaluación de eventos jurídico-administrativos ambientales desde las perspectivas neopopulista, clásica y PADDD

\begin{tabular}{|c|c|c|c|c|}
\hline EVENTO & FECHA & NEOPOPULISTA & CLÁSICA & PADDD \\
\hline Creación del parque nacional Nevado de Toluca & 1936 & 4 & 4 & 4 \\
\hline Creación de la reserva forestal Nevado de Toluca & 1937 & 0 & -2 & -2 \\
\hline $\begin{array}{l}\text { Dotación de tierras para conformar el ejido San } \\
\text { Francisco Oxtotilpan }\end{array}$ & 1939 & 4 & -4 & -4 \\
\hline $\begin{array}{l}\text { Creación de la zona protectora forestal Cuencas } \\
\text { de los Ríos Valle de Bravo, Malacatepec, Tilostoc } \\
\text { y Temascaltepec }\end{array}$ & 1941 & 0 & 2 & -2 \\
\hline $\begin{array}{l}\text { Establecimiento de veda en bosques de la } \\
\text { Cuenca de México, incluyen el Estado de México }\end{array}$ & 1947 & -4 & 4 & 2 \\
\hline Creación de Protinbos & 1969 & -4 & -4 & -2 \\
\hline $\begin{array}{l}\text { Levantamiento de veda en bosques de la } \\
\text { Cuenca de México, incluyen el Estado de } \\
\text { México }\end{array}$ & 1970 & 2 & -4 & -2 \\
\hline Creación de Probosque & 1990 & 4 & 2 & 2 \\
\hline Veda forestal en el Estado de México & $1991-1995$ & -4 & 4 & 4 \\
\hline $\begin{array}{l}\text { Cambios en la Ley General del Equilibrio } \\
\text { Ecológico y Protección al Ambiente limitan } \\
\text { aprovechamiento forestal en parques } \\
\text { nacionales }\end{array}$ & 1996 & -4 & 4 & 4 \\
\hline $\begin{array}{l}\text { Primer aprovechamiento forestal persistente } \\
\text { con un ciclo de corta de diez años en el ejido } \\
\text { San Francisco Oxtotilpan }\end{array}$ & 1997 & 4 & -4 & -4 \\
\hline $\begin{array}{l}\text { Creación del parque estatal Santuario del Agua } \\
\text { Corral de Piedra }\end{array}$ & 2003 & 0 & -2 & -2 \\
\hline $\begin{array}{l}\text { Creación del área de protección de recursos } \\
\text { naturales Valle de Bravo, Malacatepec, Tilostoc } \\
\text { y Temascaltepec }\end{array}$ & 2005 & -2 & 2 & -2 \\
\hline $\begin{array}{l}\text { Publicación del Programa de Conservación y } \\
\text { Manejo del Parque Estatal Santuario del Agua } \\
\text { Corral de Piedra }\end{array}$ & 2007 & 2 & -2 & -2 \\
\hline $\begin{array}{l}\text { Autorización del Plan de Manejo Forestal de } \\
\text { San Francisco Oxtotilpan ciclo 2008-2018 }\end{array}$ & 2008 & 4 & -4 & -4 \\
\hline $\begin{array}{l}\text { Recategorización del parque nacional Nevado } \\
\text { de Toluca a área de protección de flora y fauna }\end{array}$ & 2013 & 2 & -4 & -2 \\
\hline
\end{tabular}




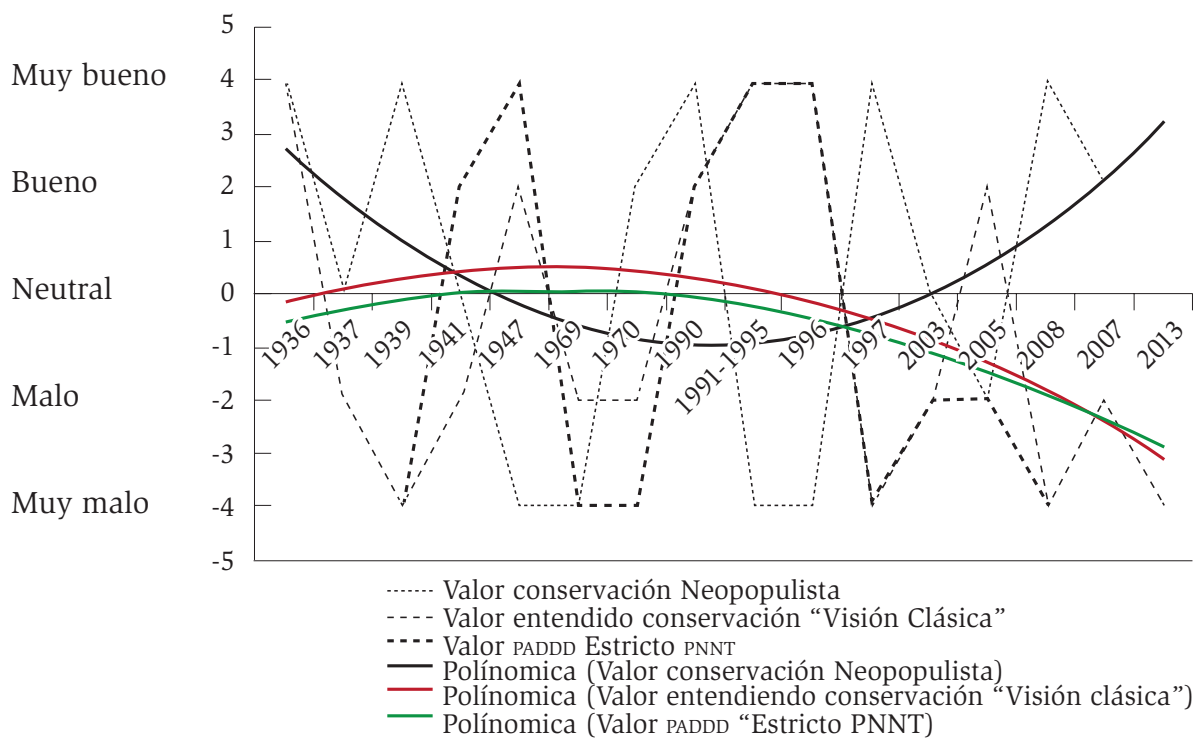

Figura 3. Situación y tendencia de la gestión de recursos forestales en San Francisco Oxtotilpan y su relación con el fenómeno PADDD

Fuente:Elaboración propia.

Como se puede observar desde las diferentes perspectivas se aprecia una discontinuidad e inclusive una fluctuación en las políticas públicas en materia forestal y de conservación que incidieron sobre el territorio de San Francisco Oxtotilpan.

Se advierte que desde las perspectivas de la conservación clásica y de la interpretación estricta del fenómeno PADDD los valores y tendencias son muy similares para el proceso de eventos jurídico-administrativos que se han producido, con una tendencia hacia la afectación negativa al ANp, sin embargo, desde la neopopulista, los valores y tendencias reflejan una percepción muy distinta, pues aunque de 1947 a 1997 se tuvieron valores negativos, las últimas acciones van aproximándose hacia valores positivos en el caso de San Francisco Oxtotilpan, sin poder generalizarlo hacia otros núcleos agrarios que se encuentran en el ANP Nevado de Toluca.

En este sentido, es importante considerar que a pesar de que la visión PADDD aparentemente sea compatible con el principio de no regresión ambiental (Peña Chacón, 2013), desde la mirada de los habitantes y propietarios colectivos 
de los recursos forestales de las Anp, en este caso la región noroeste del Nevado de Toluca, más que un retroceso en la protección y aprovechamiento de su patrimonio natural, resulta tanto un acto de justicia social, como la posibilidad de un mejor manejo para la conservación de los recursos forestales.

\section{Conclusiones}

Las políticas públicas forestales y ambientales que desde principios de siglo han repercutido en el ejido San Francisco Oxtotilpan no han presentado continuidad, ni congruencia en su formulación y aplicación, siendo muchas veces contradictorias en sus objetivos y, por tanto, han afectado, por un lado, la conservación de los bienes y servicios ambientales ofrecidos por los bosques del ANP Nevado de Toluca, y por el otro, el desarrollo de una cultura forestal que permita el manejo comunitario forestal efectivo y su avance hacia la conformación de empresas sociales forestales.

Asimismo, se puede observar que la perspectiva de análisis de estos eventos como fenómeno PADDD llega a ser parcial y cercana a la de la conservación como exclusión del uso del patrimonio natural por los habitantes locales o visión clásica, por lo que un análisis más específico sobre el efecto de las políticas públicas ambientales y de restricción al aprovechamiento del patrimonio natural de los pueblos que habitan en las ANP puede aportar una mirada alternativa y más compleja en torno a las repercusiones de la discontinuidad y cambio de políticas públicas ambientales sobre la conservación de los bienes y servicios ambientales que brindan estas áreas.

Es necesario establecer mecanismos que permitan dar continuidad y operar de manera efectiva las políticas públicas que en materia ambiental se proponen implementar para la conservación del patrimonio natural del Nevado de Toluca, pero también es menester que estas consideren la participación real y desde una visión de gobernanza de los propietarios del territorio.

Además se requiere fortalecer el capital social y la cultura forestal de los dueños de los bosques, pues hasta ahora no ha existido una política verdadera que refuerce sus capacidades para la apropiación del proceso productivo forestal, ya que históricamente, e incluso bajo las nuevas políticas forestales en las que el prestador de servicios técnicos forestales tiene un papel protagónico, 
han quedado supeditados al control por parte de agentes externos por razones político-administrativas o de conocimiento técnico.

Finalmente, el área de protección de flora y fauna Nevado de Toluca es un mosaico de sistemas socioecológicos y situaciones ambientales, por lo cual no resulta posible generalizar los hallazgos de esta investigación al ANp, los cuales son más similares a lo que acontece en la región oeste del Nevado de Toluca (Cuenca Alta del Río Balsas) que en la región este (Cuenca Alta del Río Lerma), no obstante, se aportan elementos para la discusión en cuanto a las políticas públicas a aplicar en este espacio para la conservación, así como para considerar si el cambio de decreto representa una regresión ambiental en la conservación de esta área.

\section{Fuentes consultadas}

Alcorn, J. B. (2005). "Dances around the Fire: Conservation Organizations and Community-Based Natural Resource Management”, en J. P. Brosius, A. Lowenhaupt y C. Zerner (eds.). Communities and Conservation. Histories and Politics of Community-Based Natural Resource Management. Lanham: Altamira Press, 37-68.

Barton Bray, D. y L. Merino Pérez (2005). La experiencia de las comunidades forestales en México. Veinticinco años de silvicultura y construcción de empresas forestales comunitarias. México: Secretaría de Medio Ambiente y Recursos Naturales-Instituto Nacional de Ecología/Consejo Civil Mexicano para la Silvicultura Sostenible A. C./Fundación Ford.

Bautista Calderón, L. (2007). "Las vedas forestales en el México post-revolucionario”. Tesis para obtener el grado de maestría en Estudios Regionales. México: Instituto de Investigaciones José María Luis Mora, 186 pp.

Cardeña Rodríguez, J. B. (2000). "La reactivación del sector forestal en el Estado de México. Memoria de experiencia profesional” [en línea]. Tesis para obtener el título de Ingeniero Agrónomo especialista en Bosques. Texcoco: Universidad Autónoma Chapingo-División de Ciencias Forestales. Disponible en: http://www.chapingo.mx/dicifo/tesislic/2000/ Cardena \%20Rodriguez\%20Jesus \%20B.\%202000.pdf [2014, 10 de septiembre]. 
Conafor (2011). Memoria del Encuentro Nacional de Silvicultura Comunitaria [en línea]. México: Comisión Nacional Forestal, 5 y 6 de diciembre. Disponible en: http://www.conafor.gob.mx:8080/documentos/docs/37/3789Memoria \%20del \% 20Encuentro \% 20 Nacional\% Stor202011.pdf [2015, 8 de febrero].

Conafor (2015) Silvicultura comunitaria [en línea]. Disponible en http://www. conafor.gob.mx/web/temas-forestales/silvicultura-comunitaria [2015, 10 de junio].

Conanp (2013). Estudio previo justificativo para la modificación de la declaratoria del Área Natural Protegida Parque Nacional Nevado de Toluca. México: Secretaría de Medio Ambiente y Recursos Naturales.

Conanp (2014a). Borrador a consulta del Programa de Conservación y Manejo del Área de Protección de Flora y Fauna Nevado de Toluca. México: Secretaría de Medio Ambiente y Recursos Naturales.

Conanp (2014b). Borrador a consulta del Programa de Conservación y Manejo del Área de Protección de Recursos Naturales Zona Protectora Forestal los Terrenos Constitutivos de las Cuencas de los Ríos Valle de Bravo, Malacatepec, Tilostoc y Temascaltepec. México: Secretaría de Medio Ambiente y Recursos Naturales.

FAO (2010). Evaluación de los recursos forestales mundiales 2010 [en línea], Disponible en: http://foris.fao.org/static/data/fra2010/I.pdf [2014, 8 de octubre].

Francis, G. (2008). "Evolution of contexts for protected areas governance”, en K. S. Hanna, D. A. Clark y D. S. Slocombe (eds.). Transforming Parks and Protected Areas. Nueva York: Routledge, 15-38.

Fucader (Fundación Campesina para el Desarrollo Rural) y G. García Tepexpa (2011). "Ordenamiento territorial comunitario. San Francisco Oxtotilpan, municipio de Temascaltepec, Estado de México”. Documento interno.

García, T. G. (2008). "Programa de Manejo Forestal para el Aprovechamiento de Recursos Forestales Maderables (Nivel Avanzado)”. Documento interno. Gobierno del Estado de México-Secretaría del Medio Ambiente (s/f). "Antecedentes", en "Acerca de Probosque", en Protectora de Bosques del Estado de México [en línea]. Disponible en: http://probosque.edomex.gob. $\mathrm{mx} /$ antecedentes [2014, 10 de septiembre]. 
Jujnovsky, J., L. Galván y M. Mazari-Hiriart (2013). “Zonas Protectoras Forestales: El caso de los bosques de la Cañada de Contreras, Distrito Federal”. Investigación Ambiental, 5 (2), 65-75.

Klooster, D. (2003). "Campesinos and mexican forest policy during the twentieth century”. Latin American Research Review, 38 (2), 94-126.

Klooster D. y Ambinakudige, S. (2007). "La importancia mundial del manejo forestal comunitario en México". En: Los bosques comunitarios en México. Bray D., Merino-Pérez L. y Segura-Warnholtz (2007). México: INE-Semarnat.

Manterola, C. y D. Zavando (2009). "Cómo interpretar los 'Niveles de Evidencia' en los diferentes escenarios clínicos”. Revista Chilena de Cirugía, 61 (6), 582-595.

Mascia, B., S. Pailler, R. Krithivasan, V. Roshcank, D. Burns, J. Mlotha, D. Roeber y N. Peng (2014). "Protected area downgrading, downsizing, and degazettement (PADDD) in Africa, Asia, and Latin America and the Caribbean, 1900-2010”. Biological Conservation, 169, 355-361.

Merino Pérez, L. (2004). Conservación o deterioro. El impacto de las políticas públicas en las instituciones comunitarias y en los usos de los bosques en México. México: Secretaría de Medio Ambiente y Recursos NaturalesInstituto Nacional de Ecología.

Merino Pérez, L. y G. Segura-Warnholtz (2007). "Las políticas forestales y de conservación y sus impactos en las comunidades forestales en México”, en D. Barton Bray, L. Merino Pérez y G. Segura-Warnholtz (eds.). Los bosques comunitarios en México. México: Secretaría de Medio Ambiente y Recursos Naturales-Instituto Nacional de Ecología, 77-98.

Peña Chacón, M. (dir.). (2013). El principio de no regresión ambiental en el derecho comparado latinoamericano [en línea]. San José, Costa Rica: Programa de las Naciones Unidas para el Desarrollo. Disponible en: http://www.aida-americas.org/sites/default/files/Doc_CR_Principio_ No_Regresi\%C3\%B3n_Ambiental.pdf [2014, 8 de octubre].

PNUMA (2012). GEO 5. Perspectivas del medio ambiente mundial. Medio ambiente para el futuro que queremos. Colombia: Programa de las Naciones Unidas para el Medio Ambiente.

Poder Ejecutivo (1988). "Ley General del Equilibrio Ecológico y la Protección al Ambiente”. Diario Oficial de la Federación, 28 de enero. 
Poder Ejecutivo (1991). "Decreto por el que se establece la veda temporal y parcial respecto de las especies forestales en todas sus variedades, ubicadas dentro de los límites del Estado de México". Diario Oficial de la Federación, 9 de septiembre.

Poder Ejecutivo (1995). "Decreto por el que se levanta la veda temporal y parcial dentro de los límites del Estado de México, establecida por el diverso de fecha 6 de septiembre de 1995”. Diario Oficial de la Federación, 30 de junio.

Poder Ejecutivo del Estado de México (2003). "Declaratoria del Ejecutivo del Estado por el que se establece el área Natural Protegida con la Categoría de Parque Estatal denominada 'Parque Estatal Santuario del Agua Corral de Piedra'”. Gaceta del Gobierno, 118, lunes 23 de junio.

Poder Ejecutivo del Estado de México (2007). "Resumen ejecutivo del Programa de manejo del Parque Estatal denominado 'Santuario del Agua Presa Corral de Piedra'”. Gaceta del Gobierno, 27, jueves 8 de febrero.

Porter-Bolland, L., et al. 2011. Community managed forests and forest protected areas: An assessment of their conservation effectiveness across the tropics. Forest Ecol. Manage [en línea]. [2015, 5 de marzo], doi:10.1016/j. foreco.2011.05.034.

Rights and Resources Initiative (2012). Respecting Rights, Delivering Development. Forest tenure reform since Rio 1992. Washington, D.C.: Rights and Resources Initiative.

Rockström, J. et al. (2009). "A safe operating space for humanity”. Nature, 461, 472-475.

Sánchez, A., A. Sánchez, M. García, N. Sánchez y G. García (1990). Apuntes para la historia forestal del Estado de México. Toluca: Gobierno del Estado de México-Protectora de Bosques del Estado de México.

Secretariat of the Convention on Biological Diversity (2014). Global Biodiversity Outlook 4. Montreal: Secretariat of the Convention on Biological Diversity.

Sedatu (Secretaría de Desarrollo Agrario, Territorial y Urbano) (s/f). Padrón e Historial de Núcleos Agrarios [en línea]. Disponible en: http://phina. ran.gob.mx/phina2/Sessiones [2014, 8 de octubre]. 